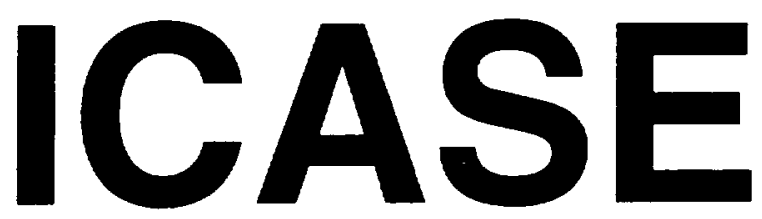

\title{
THE STRUCTURE OF VARIABLE PROPERTY, COMPRESSIBLE MIXING LAYERS IN BINARY GAS MIXTURES
}

\section{F. Kozusko, C.E. Grosch, T.L. Jackson, Christopher A. Kennedy, and Thomas B. Gatski}

NASA Contract No. NAS1-19480

May 1996

Institute for Computer Applications in Science and Engineering NASA Langley Research Center

Hampton, VA 23681-0001

Operated by Universities Space Research Association

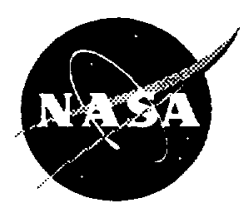

National Aeronautics and Space Administration

Langley Research Center

Hampton, Virginia 23681-0001 



\title{
The Structure of Variable Property, Compressible Mixing Layers in Binary Gas Mixtures
}

\author{
F. Kozusko \\ Department of Mathematics and Statistics \\ Old Dominion University, Norfolk, Virginia 23529 \\ C.E. Grosch \\ Departments of Oceanography and Computer Science \\ Old Dominion University, Norfolk, Virginia 23529 \\ T.L. Jackson \\ Institute for Computer Applications in Science and Engineering \\ NASA Langley Research Center, Hampton, VA 23681-0001 \\ Christopher A. Kennedy \\ Sandia National Laboratory \\ Combustion Research Facility, Livermore, CA 94551-0969 \\ Thomas B. Gatski \\ Aerodynamic and Acoustic Methods Branch \\ NASA Langley Research Center, Hampton, VA 23681-0001
}

\begin{abstract}
We present the results of a study of the structure of a parallel compressible mixing layer in a binary mixture of gases. The gases included in this study are hydrogen $\left(\mathrm{H}_{2}\right)$, helium ( $\mathrm{He}$ ), nitrogen $\left(\mathrm{N}_{2}\right)$, oxygen $\left(\mathrm{O}_{2}\right)$, neon $(\mathrm{Ne})$ and argon $(\mathrm{Ar})$. Profiles of the variation of the Lewis and Prandtl numbers across the mixing layer for all thirty combinations of gases are given. It is shown that the Lewis number can vary by as much as a factor of eight and the Prandtl number by a factor of two across the mixing layer. Thus assuming constant values for the Lewis and Prandtl numbers of a binary gas mixture in the shear layer, as is done in many theoretical studies, is a poor approximation. We also present profiles of the velocity, mass fraction, temperature and density for representative binary gas mixtures at zero and supersonic Mach numbers. We show that the shape of these profiles is strongly dependent on which gases are in the mixture as well as on whether the denser gas is in the fast stream or the slow stream.
\end{abstract}

The first three authors were supported by the National Aeronautics and Space Administration under NASA Contract No. NAS1-19480 while in residence at the Institute for Computer Applications in Science and Engineering (ICASE). NASA Langley Research, Hampton, VA 23681-0001. 



\section{Introduction}

In the last decade the structure and stability of compressible mixing layers has become, once again, an area of very active research. There are many experimental studies (Brown and Roshko ${ }^{[1]}$; Bogdanoff ${ }^{[2]}$; Papamoschou and Roshko ${ }^{[3]}$; Samimy and Elliot ${ }^{[4]}$; Elliot and Samimy ${ }^{[5]}$; Clemens and Mungal ${ }^{[6]}$; Hall, et al. ${ }^{[7]}$; Elliot, et al. ${ }^{[8]}$ ) of the overall structure and the turbulence, principally the large scale structures, of these flows. In some of these experiments, for example those of Papamoschou and Roshko ${ }^{[3]}$, Hall, et al. ${ }^{[7]}$ and Ninnemann and $\mathrm{Ng}^{[9]}$, different gases are in the two streams. In other words, the mixing layer exists in a binary gas mixture.

There are also a substantial number of theoretical and numerical studies of the structure of the compressible mixing layer as well as the stability of this flow. In almost all cases, it was assumed that the fluid properties in the mixing layer were those of a single gas and that variations of density and viscosity across the layer were due solely to the variation in the temperature. Of course as the mixture fractions of the gases change across the mixing layer, the viscosity, diffusivity, specific heat, etc of the mixture will change so as to reflect the changing composition of the mixture as well as the change in the temperature.

Jackson and Grosch ${ }^{[10]}$ formulated the stability problem of a compressible mixing layer for a gas mixture, assuming that the mean flow field was known. They showed that the dimensionless sonic speeds of the two streams as well as the convective Mach number were dependent on the composition of the gas mixture. Kennedy and Gatski ${ }^{[11]}$ carried out a theoretical calculation of the structure of a supersonic/supersonic mixing layer in the binary gas mixture resulting from the mixing of a hydrogen stream with a nitrogen stream. Calculations were carried out for five values of the convective Mach number. The Kennedy-Gatski study included the variation of the fluid properties across the mixing layer caused by the variation of the gas composition. The purpose of this paper is, in part, to correct and, in part, to extend to a wider variety of binary gas mixtures the study of Kennedy and Gatski ${ }^{[11]}$. In particular, we will show that certain of their results are incorrect and certain of their conclusions must be modified.

The gases included in this study are hydrogen $\left(\mathrm{H}_{2}\right)$, helium $(\mathrm{He})$, nitrogen $\left(\mathrm{N}_{2}\right)$, oxygen $\left(\mathrm{O}_{2}\right)$, neon $(\mathrm{N} \epsilon)$ and argon $(\mathrm{Ar})$. The choice of gases was not arbitrary. Hydrogen is the fuel for proposed scram-jet engines. Papamoschou and Roshko ${ }^{[3]}$ and Hall, et al. ${ }^{[7]}$ used binary combinations of $\mathrm{He}, \mathrm{N}_{2}$ and $\mathrm{Ar}$ in their experiments. An accurate calculation of the structure of flow field in a gas mixture and a meaningful comparison of the results of theory with experiment must incorporate not only the variation of density with composition but also the changes with composition and temperature of the thermal properties and the transport properties of the mixture .

\section{Thermal Properties and Transport Coefficients}

The binary gas mixture is characterized by the mass fractions, $\left\{F_{j}\right\}, j=1,2$ of the species. The mixture properties, heat capacity $\left(C_{P}\right)$, coefficient of viscosity $(\mu)$, thermal conductivity $(\kappa)$ 
and binary diffusion coefficient $\left(D_{12}\right)$ are functions of the $\left\{F_{j}\right\}$ and of the temperature $T$. These properties vary continuously through the mixing layer as the temperature and mass fractions vary.

The quest for an understanding of the variation of these thermal and transport properties with temperature and mixture composition has been the central topic of the Kinetic Theory of Gases and has engendered hundreds, if not thousands, of experiments for well over a century and a half (Chapman and Cowling ${ }^{[12]}$; Hirschfelder, et al. ${ }^{[13]}$; Touloukian, et al. ${ }^{[14]}$; Maitland, et al. $\left.{ }^{[15]}\right)$. Indeed, Chapman and Cowling's book, "The Mathematical Theory of Non-Uniform Gases", is subtitled "An Account of the Kinetic Theory of Viscosity, Thermal Conduction and Diffusion in Gases". Despite the vast effort which has been expended on this topic, the theory is far from complete and yields some seemingly paradoxical results.

A satisfactory theory for the variation of transport properties of monatomic gases, both in the unmixed state and as a mixture, has been developed (Chapman and Cowling ${ }^{[12]}$; Hirschfelder, et al. ${ }^{[13]}$; Touloukian, et al. ${ }^{[14]}$; Maitland, et al..$\left.^{[5]}\right)$ in terms of the molecular scattering cross sections, temperature and composition. For binary mixtures the theory is reasonably straightforward but becomes much more complex for mixtures of more than two gases. There is good agreement between theory and experiment for binary gas mixtures. However, there is much less experimental conformation of the theory for other than binary mixtures.

The theory of the transport properties of mixtures of monatomic and polyatomic gases and of binary mixtures of polyatomic gases is in a far less satisfactory state. The basic kinetic theory for the transport properties of a pure polyatomic gas is a semi-classical one with certain quantum mechanical corrections (Touloukian, et al. ${ }^{[14]}$; Maitland, et al. ${ }^{[15]}$ ). It appears that there is a great sensitivity of the results to the detailed structure, including the anisotropy, of the molecular potentials of polyatomic gases and these are not well known. In addition, some of the collisions are inelastic or partially elastic and this adds further difficulties. For mixtures, the theory is even more complicated and is, at best, no more accurate than the first order Chapman-Cowling approximations and usually less accurate because of other approximations which must be made in order to make the theory useful. The theory must be supplemented by experimental measurements, say of the variation of viscosity with temperature, in order to obtain the values of intermolecular potentials required to calculate the other transport coefficients. These experimental measurements are, in many cases, lacking or inadequate.

In the absence of a complete theory, we have used a mixture of the results of experimental measurements and approximate theory to obtain the thermal properties and transport coefficients of the binary gas mixtures.

\subsection{Heat Capacity}

The thermodynamic properties of a large number of substances over a wide range of tem-

peratures are tabulated by McBride, et al. ${ }^{[16]}$. The heat capacity of the individual gases used in this study were taken from this tabulation and stored, as a function of temperature, in tabular form within the code. The values for the individual gases, as a function of temperature, were 
obtained by interpolation. The mixture $C_{P}$ was calculated from :

$$
C_{P}=F_{1} C_{P_{1}}+F_{2} C_{P_{2}} \text {. }
$$

\subsection{Coefficient of Viscosity}

The viscosity of the individual gases was calculated using the first order Kinetic Theory formulae as given by Maitland, et al. ${ }^{[15]}$ (see their Appendix A5.2) and also by Anderson ${ }^{[17]}$. The viscosity is an explicit function of temperature and of a collision integral which, in turn, is also a function of temperature. Maitland, et al. ${ }^{[5]}$ tabulate values of various collision integrals (see their Appendix 2) as a function of temperature. We used the values for the Lennard-Jones (12-6) potential given in table A2.2. The calculated viscosity of the individual gases was stored within the code in tabular form as a function of temperature. The value of the viscosity at a specific temperature was obtained by interpolation.

The viscosity of a binary mixture of monatomic gases is given by Maitland, et al. ${ }^{[15]}$ as a function of the mole fractions of the gases, the viscosities of the individual gases and of a so-called interaction viscosity arising from collisions between the molecules of the different gases. We converted the mole fractions to mass fractions and used the values of the Lennard-Jones (12-6) collision integrals in the calculation of the interaction viscosity. Again, the values of interaction viscosity were stored within the code in tables as a function of temperature. Interpolation was used to obtain the value at a specific temperature. The value of the mixture viscosity at a specific temperature was obtained using the formulae of Maitland, et al. ${ }^{[15]}$ with the $\left\{F_{j}\right\}$ of the individual gases, their viscosities and the interaction viscosity.

It should be noted that the theory used was that for a binary mixture of monatomic gases. As pointed out by Maitland, et al. ${ }^{[15]}$ (see page 315 and following) at first order the form of the equations for the viscosity of a binary gas mixture of polyatomic gases is the same as for a binary mixture of monatomic gases. For this reason, we used the formulae for monatomic gases to also calculate the viscosities of polyatomic gases. Comparison of the calculated viscosities for the polyatomic gases with tabulated experimental results (Touloukian, et al. ${ }^{[14]}$ ) showed good agreement; the differences ranged from $0.01 \%$ to $0.20 \%$.

\subsection{Thermal Conductivity}

We attempted to use a theory, similar to that used for viscosity, to calculate the thermal conductivity. The calculated values were in very poor agreement with tabulated experimental data for the polyatomic gases $\left(\mathrm{H}_{2}, \mathrm{~N}_{2}\right.$ and $\left.\mathrm{O}_{2}\right)$. This lead us to use the tabular experimental data (Touloukian, et al. ${ }^{[18]}$ ) for the thermal conductivity of the individual gases. The mixture conductivity was then calculated using the method of Mason and Saxena recommended by

Touloukian, et al. ${ }^{[18]}$. Because this theory has one form for mixtures of monatomic gases and one for mixtures of polyatomic gases, the polyatomic form was used if at least one of the gases was polyatomic. 


\subsection{Binary Diffusion Coefficient}

Maitland, et al. ${ }^{[15]}$ give a formula from first order Kinetic Theory for the binary diffusion coefficient, $D_{12}$ of a gas mixture. This is a function of temperature and involves a collision integral which is also a function of temperature. In contrast to mixture specific heat, viscosity and thermal conductivity, $D_{12}$ is independent of the mass fractions of the gases. There is relatively little experimental data for $D_{12}$. We compared the computed values with the experimental values given by Hirschfelder, et al. ${ }^{[13]}$ and by Bzowski, et al. ${ }^{[19]}$. The agreement was found to be satisfactory; in most cases differences were in the range of $1 \%$ to $2 \%$ with the maximum difference being $3.3 \%$. As with the other coefficients, $D_{12}$ was computed at a series of temperatures for each combination of gases. These values were stored in tabular form and values at specific temperatures were found by interpolation.

\section{Basic Equations}

In what follows in referring to a binary gas, $G a s_{1}-G a s_{2}$, we use the convention that $G a s_{1}$ has a mass fraction $F_{1}$ which is zero at $+\infty$ and that $G a s_{2}$ has a mass fraction $F_{2}$ which is zero at $-\infty$. That is, the stream at $-\infty$ is entirely $G a s_{1}$ and that at $+\infty$ is entirely $G_{a s}$. These gases have molecular weights $W_{1}$ and $W_{2}$, with $W=W_{2} / W_{1}$ being their ratio. The velocity field has components $(U, V), T$ is the temperature and $\rho$ is the density.

The equations were made nondimensional by the values at $+\infty$ of the density, $\rho_{\infty}$, temperature, $T_{\infty}$, speed, $U_{\infty}$, pressure, $P_{\infty}=\mathcal{R} \rho_{\infty} T_{\infty} / W_{2}$ ( $\mathcal{R}$ is the universal gas constant ), viscosity, $\mu_{\infty}$, thermal conductivity, $\kappa_{\infty}$, mass diffusion coefficient, $D_{12, \infty}$, and specific heat, $C_{P, \infty}$. The ratio of specific heats at $+\infty$ is $\gamma_{\infty}$ and $M_{\infty}=U_{\infty} / a_{\infty}$ is the Mach number at $+\infty$ with the speed of sound given by

$$
a_{\infty}^{2}=\left(\gamma_{\infty}-1\right) C_{P \infty} T_{\infty}
$$

The Prandtl and Lewis numbers are defined as usual

$$
\operatorname{Pr}=\frac{\mu C_{P}}{\kappa}
$$

and

$$
L e=\frac{\kappa}{\rho D_{12} C_{P}} .
$$

These vary across the shear layer because the transport coefficients and thermal properties vary across the layer.

Using the Howarth-Dorodnitsyn transformation,

$$
Y=\int_{0}^{y} \rho d y, \quad \hat{V}=\rho V+U \int_{0}^{y} \rho_{x} d y,
$$

and the boundary layer approximation, with primes denoting differentiation with respect to the similarity variable,

$$
\eta=\frac{Y}{2 \sqrt{x}},
$$


and with

$$
U=f^{\prime}(\eta), \quad \hat{V}=\left(\eta f^{\prime}-f\right) / \sqrt{x}
$$

the nondimensional equations for the steady, laminar flow of compressible binary mixture are,

$$
\begin{gathered}
\left(\rho \mu f^{\prime \prime}\right)^{\prime}+2 f f^{\prime \prime}=0 \\
\left(\left(\frac{\rho \mu}{P r}\right) C_{P} T^{\prime}\right)^{\prime}+2 C_{P} f T^{\prime}+\left(\gamma_{\infty}-1\right) M_{\infty}^{2} \rho \mu\left(f^{\prime \prime}\right)^{2}+\left(\frac{\rho \mu}{\operatorname{Pr} L e}\right) T^{\prime} \sum_{j=1}^{2} C_{P, j} F_{j}^{\prime}=0 \\
\left(\left(\frac{\rho \mu}{\operatorname{Pr} L e}\right) F_{1}^{\prime}\right)^{\prime}+2 f F_{1}^{\prime}=0 \\
1=F_{1}+F_{2}, \\
1=\rho T\left(W F_{1}+F_{2}\right), \\
C_{P}=C_{P_{1}} F_{1}+C_{P_{2}} F_{2} .
\end{gathered}
$$

The boundary conditions are

$$
\begin{gathered}
f^{\prime}=T=F_{2}=1, \quad F_{1}=0 \quad \eta \rightarrow+\infty, \\
f^{\prime}=\beta_{U}, \quad T=\beta_{T}, \quad F_{1}=1, \quad F_{2}=0 \quad \eta \rightarrow-\infty .
\end{gathered}
$$

The parameters $\beta_{U} \in[0,1)$ and $\beta_{T}>0$ are the ratios of the speed and temperature of the gas stream at $-\infty$ to that at $+\infty$. If $\beta_{T}$ is less than one the gas in the slow stream is colder than the gas in the fast stream. If $\beta_{T}$ is greater than one the converse is true. The remaining parameter is $W$, the ratio of molecular weights. If $W>1$ the gas in the fast stream is heavier than that in the slow stream, and if $W<1$ the gas in the fast stream is lighter than that in the slow stream.

The equations given by Kennedy and Gatski[11] differ from those above because of their inconsistency in defining the Lewis number. Their definition of the Lewis number (just after equation (5) on page 664) is the same as that used here and they used this definition in calculating the Lewis number. However, in their equation (5) the Lewis number is the reciprocal of that defined. Their equations (18) through (22) perpetuate this inconsistency. In addition, they used the kinetic theory for mixtures of monatomic gases (even though both $\mathrm{H}_{2}$ and $\mathrm{N}_{2}$ are polyatomic) to calculate the transport coefficients. They then used an ad hoc scheme to correct the thermal conductivity to account for polyatomic effects. In contrast, we here use a combination of the theory for polyatomic gases and tabulated experimental data.

The system of equations for $f, T$ and $F_{1}$ is seventh order and there are only six boundary conditions. A seventh boundary condition can be found by matching the free stream pressures across the layer (Ting ${ }^{[20]}$ ) if at least one of the streams is supersonic. However, if both are subsonic, Klemp and Acrivos ${ }^{[21]}$ showed that this condition is incomplete. The seventh boundary condition is equivalent to determining the value of the stream function $f$ at $\eta=0$. We have set $f(0)=0$.

The system of equations and boundary conditions is a two point boundary value problem which can be solved by a shooting method. In the limit $\eta \rightarrow-\infty$ the asymptotic forms of 
(6) - (8) can be integrated exactly. Using the three boundary conditions for $f^{\prime}(-\infty), T(-\infty)$ and $F_{1}(-\infty)$ to determine three of the constants of integration, these solutions contain four unknown constants. Choosing values for these four constants determines the complete initial conditions for (6)-(8) at $\eta=-\infty$. A fourth order double precision Runge-Kutta method was used to integrate the system to $\eta=\infty$. Iteration on the values of the four constants was carried on until the condition at $\eta=0$ and the boundary conditions at $\eta=\infty$ are satisfied. The system was found to be extremely stiff and a nested iteration scheme was used. In this scheme, one condition at a time was used and after each was satisfied additional iterations were required to resatisfy the previously used conditions. This iterative process was continued until all of the boundary conditions were satisfied.

\section{Results}

Kennedy and Gatski ${ }^{[11]}$ only considered the mixture $\mathrm{N}_{2}-\mathrm{H}_{2}$. Here we considered the thirty different combinations of $\mathrm{H}_{2}, \mathrm{He}, \mathrm{Ne}, \mathrm{N}_{2}, \mathrm{O}_{2}$ and $\mathrm{Ar}$ for $\mathrm{Gas}_{1}-\mathrm{Gas}_{2}$. We did not consider systems of the same gas in both streams because this would require the inclusion of self-diffusion for consistency and the theory and experimental measurements for this effect are wanting. Table 1 contains the values of $W$ for each combination. This parameter has a wide range, from 19.813 for a $\mathrm{H}_{2}-\mathrm{Ar}$ mixture to 0.050 for a $\mathrm{Ar}-\mathrm{H}_{2}$ mixture and there is a corresponding variation in the structure of the mixing layer. We first present the variation of the Lewis and Prandtl numbers across the shear layer at $M_{\infty}=0$. In all cases we took $T_{\infty}=300^{\circ} \mathrm{K}, \beta_{U}=0.5$ and $\beta_{T}=1.5$.

Figures 1, 2 and 3 are plots of the variation of the Lewis number across the mixing layer. For most pairs of gases there are relatively large variations in the Lewis number. The largest variation occurs for mixtures of $\mathrm{He}$ with $\mathrm{Ar}, \mathrm{O}_{2}$ and $\mathrm{N}_{2}$ with the ratio of the values at $\pm \infty$ for $H \epsilon-A r$ and $A r-H \epsilon$ being about eight. Large variations in the value of the Lewis number also occur for mixtures of $\mathrm{H}_{2}$ with $\mathrm{Ar}, \mathrm{O}_{2}$ and $\mathrm{N}_{2}$. It should be noted that the magnitude of the variation is not strictly monotone with molecular weight ratio. The values of $W$ are larger for combinations of $\mathrm{H}_{2}$ with the heavier gases than for $\mathrm{He}$ and heavy gases. Yet the variation of the Lewis number is smaller for combinations of $\mathrm{H}_{2}$ with heavy gases than for $\mathrm{He}$ with heavy gases. A few gas mixtures have nearly constant Lewis number; mixtures of $\mathrm{O}_{2}, \mathrm{~N}_{2}$ and $\mathrm{Ar}$ as well as the $\mathrm{H}_{2}-\mathrm{He}$ and $\mathrm{He}-\mathrm{H}_{2}$ mixtures. It might seem that a nearly constant Lewis number would occur only for mixtures with $W$ close to one. This is not true. The molecular weight ratio for mixtures of $\mathrm{O}_{2}, N_{2}$ and $A r$ lies in the range of 0.8 to 1.2 , and these mixtures have relatively constant Lewis number across the layer. However, mixtures of of $\mathrm{H}_{2}$ and $\mathrm{He}$ have $\mathrm{W}$ close to 2.0 (or 0.5 ) and still have small variation of the Lewis number across the layer. Finally, it can be seen that the Lewis number profiles are generally not symmetric about the centerline of the mixing layer and that interchanging the gases between $+\infty$ and $-\infty$ does not yield mirror images about the mixing layer centerline. Particularly noticeable cases are mixtures of $\mathrm{H}_{2}$ or $\mathrm{He}$ with any of $\mathrm{Ar}, \mathrm{Ne}, \mathrm{N}_{2}$ or $\mathrm{O}_{2}$.

The variation of the Prandtl number across the mixing layer is shown in Figures 4,5 and 
$6 \mathrm{a}, 6 \mathrm{~b}$ for all of the gas mixtures. Figure 4 is a plot of the Prandtl number for the same gas combinations as those whose Lewis number variation is shown in Figure 1. Figures 5 and 2 have a similar relation. However, for the gases whose Lewis number variation is shown in Figure 3, a single figure could not adequately display the Prandtl number variation. As a result, Figure 6 has two parts (a) and (b). There is a substantial variation of the Prandtl number across the mixing layer for all combinations of $\mathrm{H}_{2}$ or $\mathrm{He}$ with the heavier gases $\mathrm{Ar}, \mathrm{Ne}, \mathrm{N}_{2}$ ог $\mathrm{O}_{2}$. As with the Lewis number variation, the Prandtl number curves are generally not symmetric about the center of the mixing layer, nor does interchanging the gases between $+\infty$ and $-\infty$ yield mirror images of the Prandtl number curves.

In comparing these results to those of Kennedy and Gatski ${ }^{[11]}$ there is general disagreement between the results for the Prandtl and Lewis numbers given here and those of Kennedy and Gatski. The shapes of the Prandtl and Lewis number profiles given here are, approximately, "mirror images" about $\eta=0$ of those given by Kennedy and Gatski. The Prandtl number is 0.700 in the slow $N_{2}$ stream, but only about 0.655 at $\eta=-20.0$. Its value decreases slowly towards the center of the shear layer, reaching a minimum of about 0.38 just short of the middle of the shear layer and then rises very rapidly with distance beyond the center of the shear layer, reaching a value of 0.711 in the fast $H_{2}$ stream. The Lewis number distribution is, on the other hand, a monotone function of distance through the shear layer. It rises slowly from a value of 0.322 in the $N_{2}$ stream until near the center of the shear layer and then asymptotes over a short distance to 2.010 in the $\mathrm{H}_{2}$ stream. The different shape of the profiles of these parameters between our results and those of Kennedy and Gatski is due to the inconsistent definitions used by Kennedy and Gatski as discussed in Section 3.

Finally, the lack of a reflection symmetry of both the Lewis and Prandtl number curves about the center of the mixing layer is a result of the non-symmetric variation of the temperature across the layer. As stated above, all of the transport coefficients are functions of temperature and so show a corresponding non-symmetric variation across the layer. The "tails" of the Lewis number and Prandtl number curves are always longest on the side of the mixing layer where a lighter gas is diffusing into the higher temperature region as compared to the reverse situation. A good example of this is provided by the Lewis number and Prandtl number curves for $\mathrm{Ar}-\mathrm{H}_{2}$ as compared to those for $\mathrm{H}_{2}-\mathrm{Ar}$.

We next present additional results for the case considered by Kennedy and Gatski ${ }^{[1]}, \mathrm{N}_{2}-\mathrm{H}_{2}$ for which $W=0.072$. For this case the slow speed stream consists of $N_{2}$ and the high speed stream is $\mathrm{H}_{2}$. Figure 7 shows profiles of the dimensionless velocity, mass fractions, temperature and density as a function of the dimensionless distance across the shear layer for $M_{\infty}=0$. The general shapes of the velocity, mass fraction, temperature and density profiles are similar to those obtained by Kennedy and Gatski. This is, in part, because the values of these variables at $\pm \infty$ are set by the boundary conditions and the equations enforce a smooth transition between these boundary values. As seen from Figure 7, the velocity profile has a modest "tail" into the slow stream and a rapid transition to the fast stream. The mass fraction of $\mathrm{H}_{2}$ decreases quite slowly on the slow speed side of the mixing layer $(\eta \rightarrow-\infty)$; there is substantial diffusion of the $\mathrm{H}_{2}$ from the relatively cold fast stream into the the relatively hot slow stream. This is also clear 
from the Lewis and Prandtl number profiles shown in Figures 3 and 6(a). This effect is also apparent in the temperature and density profiles which have very long "tails" on the $\mathrm{N}_{2}$ side of the layer; thus the temperature and density profiles are shifted towards the $N_{2}$ side of the layer as compared to the velocity profile which lies almost exactly in the center of the layer. The mid-points of the mass fraction profiles are shifted towards the $N_{2}$ side of the layer although not as much as the density and temperature profiles. Our results are similar to, but not identical to those of Kennedy and Gatski. We also find that one of the points made by Kennedy and Gatski is still valid; assuming constant values for the Lewis and Prandtl numbers in the shear layer is a poor approximation when considering binary gases.

Next, the corresponding profiles, for $M_{\infty}=0$, in the case of $H_{2}-N_{2}(W=13.897)$ is shown in Figure 8. In this case the slow speed stream is $H_{2}$ and the high speed stream is $N_{2}$. Note that Kennedy and Gatski ${ }^{[11]}$ did not present any results for this case. The mean profiles of velocity, mass fraction, temperature and density are quite different from those of the $\mathrm{N}_{2}-\mathrm{H}_{2}$ case. In this case the velocity and mass fraction profiles are those of a very thin shear layer. The temperature and density profiles indicate an only slightly thicker layer with short "tails" in the higher speed, colder $N_{2}$ stream. Again this behavior is seen in the Lewis and Prandtl number profiles of Figures 3 and 6 (b). One can see that there is relatively little diffusion of $\mathrm{H}_{2}$ from the hotter, slower stream into the $N_{2}$ of the colder, faster stream. Note that for this case, in contrast to the $\mathrm{N}_{2}-\mathrm{H}_{2}$ flow, the density and temperature profiles have only a minimal offset from the velocity and mass fraction profiles. The centers of all of the profiles are slightly shifted towards the high speed, $\mathrm{N}_{2}$ side of the layer

Finally, the velocity, mass fractions, temperature and density profiles, at a free stream Mach number of 3, for $\mathrm{N}_{2}-\mathrm{H}_{2}$ are shown in Figure 9 and for $\mathrm{H}_{2}-\mathrm{N}_{2}$ in Figure 10. The mean profiles of velocity, mass fraction and density at this higher Mach number are nearly identical to those at $M=0$ shown in Figures 7 and 8. This is true for both the case in which $N_{2}$ is in the slow stream and $H_{2}$ is in the fast stream (Figure 9) as well as the reverse (Figure 10). The only exception is the temperature profile for the $\mathrm{N}_{2}-\mathrm{H}_{2}$ case. The long tail extending into the slow speed, $N_{2}$ side of the mixing layer is quite different at $M=3$ as compared to $M=0$. With $M=3$, the temperature gradient is considerably higher near the center of the layer than at $M=0$. However, there is almost no difference in the temperature profile between $M=0$ and $M=3$ for the case in which $H_{2}$ is in the slow stream and $N_{2}$ is in the fast stream.

\section{Conclusions}

By calculating the variation of the Lewis and Prandtl numbers across a mixing layer for all thirty binary combinations of $\mathrm{H}_{2}, \mathrm{He}, \mathrm{N}_{2}, \mathrm{O}_{2}, \mathrm{Ne}$ and $\mathrm{Ar}$, we have shown that for most pairs of gas mixtures there are relatively large variations in the Lewis number across the mixing layer. There are also large variations in the Prandtl number for some of these binary gas mixtures. The largest variation of both the Lewis and Prandtl numbers occurs for mixtures of the light gases $\mathrm{He}$ or $\mathrm{H}_{2}$ with the heavy gases $\mathrm{Ar}, \mathrm{O}_{2}$ and $\mathrm{N}_{2}$. However, the magnitude of the variation is not

strictly monotone with the molecular weight ratio. Both the Lewis and Prandtl number curves 
are not symmetric about the center of the mixing layer. This is a result of the non-symmetric variation of the temperature across the layer. All of the transport coefficients are functions of temperature and so show a corresponding non-symmetric variation across the layer. The "tails" of the Lewis number and Prandtl number curves are always longest on the side of the mixing layer where a lighter gas is diffusing into the higher temperature region as compared to the reverse situation. Assuming constant values for the Lewis and Prandtl numbers of a binary gas mixture in the shear layer is a poor approximation.

The general shapes of the velocity, mass fraction, temperature and density profiles for a binary mixture of $\mathrm{N}_{2}$ in the slow stream and $\mathrm{H}_{2}$ in the fast stream are similar to those obtained by Kennedy and Gatski ${ }^{[11]}$ despite their use of an inconsistent definition of the Lewis number. This is, in part, because the values of these variables at $\pm \infty$ are set by the boundary conditions and the equations enforce a smooth transition between these boundary values. The temperature and density profiles are shifted towards the $N_{2}$ side of the layer as compared to the velocity profile. For the opposite case of $\mathrm{H}_{2}$ in the slow stream and $\mathrm{N}_{2}$ in the fast stream (not done by Kennedy and Gatski) the profiles of velocity, mass fraction, temperature and density are very different from those of the other case. Here the velocity and mass fraction profiles are those of a very thin shear layer. The temperature and density profiles are those of a slightly thicker layer with quite short "tails" in the higher speed, colder $N_{2}$ stream. There is relatively little diffusion of $\mathrm{H}_{2}$ from the hotter, slower stream into the $\mathrm{N}_{2}$ of the colder, faster stream. For this case, in contrast to the $\mathrm{N}_{2}-\mathrm{H}_{2}$ flow, the density and temperature profiles have only a minimal offset from the velocity and mass fraction profiles. Hence, one would expect that interchanging the gases in the slow and high speed streams would have a considerable effect on the flow stability and mixing. Finally, for the conditions used in this calculation, the profiles at $M=3$ are very similar to those for $M=0$. 
Table 1: The ratio of molecular weights $W$, defined as the molecular weight of the gas at $\eta=+\infty$ divided by the molecular weight of the gas at $\eta=-\infty$, for the different gases considered in this study. The top row corresponds to the gases in the freestream at $\eta=+\infty$, while the first column corresponds to the gases in the freestream at $\eta=-\infty$.

\begin{tabular}{|c||c|c|c|c|c|c|}
\hline & $\mathrm{H}_{2}$ & $\mathrm{He}$ & $\mathrm{Ne}$ & $\mathrm{N}_{2}$ & $\mathrm{O}_{2}$ & $\mathrm{Ar}$ \\
\hline \hline $\mathrm{H}_{2}$ & 1.000 & 1.986 & 10.011 & 13.897 & 15.873 & 19.813 \\
\hline $\mathrm{He}$ & 0.504 & 1.000 & 5.042 & 6.999 & 7.994 & 9.979 \\
\hline $\mathrm{Ne}$ & 0.100 & 0.198 & 1.000 & 1.388 & 1.585 & 1.979 \\
\hline $\mathrm{N}_{2}$ & 0.072 & 0.143 & 0.720 & 1.000 & 1.142 & 1.426 \\
\hline $\mathrm{O}_{2}$ & 0.063 & 0.125 & 0.631 & 0.876 & 1.000 & 1.248 \\
\hline $\mathrm{Ar}$ & 0.050 & 0.100 & 0.505 & 0.701 & 0.801 & 1.000 \\
\hline
\end{tabular}




\section{References}

[1] G.L. Brown and A. Roshko, "On density effects and large structure in turbulent mixing layers", J. Fluid Mechanics, 64, 775 (1974).

[2] D.W. Bogdanoff, "Compressibility effects in turbulent shear layers", AIAA J., 21, 926 (198.3).

[3] D. Papamoschou and A. Roshko, The compressible turbulent shear layer: An experimental study. J. Fluid Mechanics, 197:453-47, 1988.

[4] M. Samimy and G.S. Elliot, Effects of compressibility on the characteristics of free shear layers. AIAA J., 28(3):439-445, 1990.

[5] G.S. Elliot and M. Samimy, "Compressibility effects in free shear layers", Phys. Fluids A, 2, 1231 (1990).

[6] N.T. Clemens and M.G. Mungal. "Two and three dimensional effects in the supersonic mixing layer", AIAA J., 30, 3 (1992).

[7] J.L. Hall, P.E. Dimotakis and H. Rosemann, "Experiments in non-reacting compressible shear layers study", AIAA Paper 91-0629 (1991).

[8] G.S. Elliot, M. Samimy and S.A. Arnette, "The characteristics and evolution of large-scale structures in compressible mixing layers", Phys. Fluids, 7, 864 (1995).

[9] T.A. Ninnemann and W.F. Ng, "A concentration probe for the study of mixing in supersonic shear flows", Exp. Fluids, 13, 98 (1992).

[10] T.L. Jackson and C.E. Grosch, "Absolute/convective instabilities and the convective Mach number in a compressible mixing layer", Physics of Fluids A, 6, 949 (1990).

[11] C.A. Kennedy and T.B. Gatski, "Self-similar supersonic variable-density shear layers in binary systems", Phys. Fluids, 6, 662 (1994).

[12] S. Chapman and T.G. Cowling, The Mathematical Theory of Non-Uniform Gases (Cambridge University Press, 1970).

[13] J.O. Hirschfelder, C.F. Curtiss and R.B. Bird, Molecular Theory of Gases and Liquids (John Wiley and Sons, 1954).

[14] Y.S. Touloukian, S.C. Saxena and P. Hestermans, Thermophysical Properties of Matter. Volume 11: Viscosity (Plenum Press 1975).

[15] G.C. Maitland, M. Rigby, E.B. Smith and W.A. Wakeham, Intermolecular Forces (Their Origin and Determination) (Clarendon Press, Oxford 1981). 
[16] B.J. McBride, S. Heimel, J.G. Ehlers and S. Gordon, "Thermodynamic properties to $6000^{\circ} \mathrm{K}$ for 210 substances involving the first 18 elements", NASA SP-3001 (1963).

[17] J.D. Anderson, Jr, Hypersonic and High Temperature Gas Dynamics (McGraw-Hill, 1989).

[18] Y.S. Touloukian. P.E. Liley and S.C. Saxena, Thermophysical Properties of Matter. Volume 3: Thermal Conductivity (Plenum Press, 1970).

[19] J. Bzowski, J. Krestin, E.A. Mason and F.J. Uribe, "Equilibrium and Transport Properties of Gas Mixtures at Low Density", J. Phys. Chem. Ref. Data, 19, 1179 (1990).

[20] L. Ting, "On the Mixing of Two Parallel Streams", J. Math. Phys., 28, 153 (1959).

[21] J.B. Klemp and A. Acrivos, "A Note on the Laminar Mixing Layer of Two Uniform Parallel Semi-infinite Streams", J. Fluid Mech., 55, 25 (1972). 


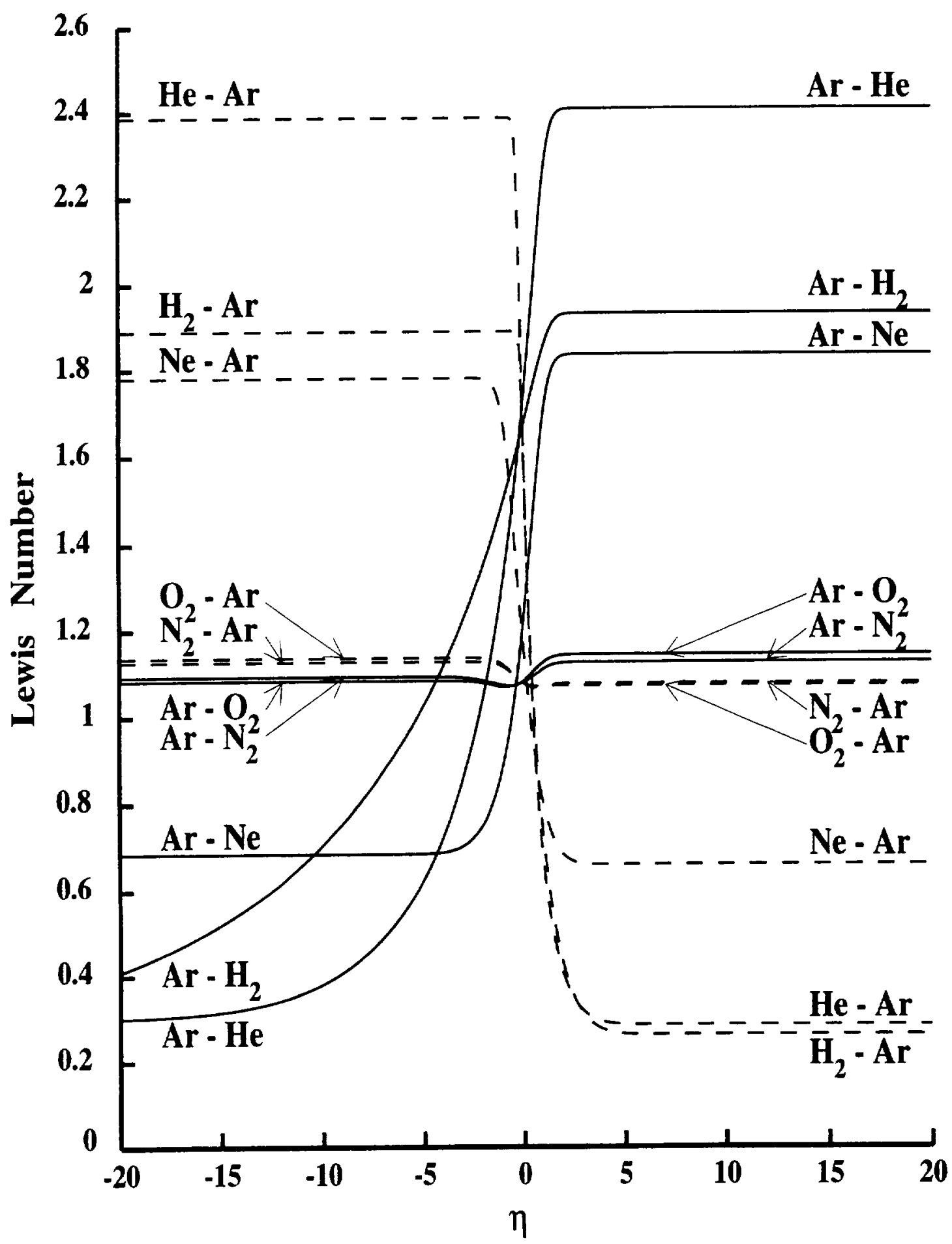

Figure 1. Plot of the Lewis number as a function of $\eta$ for $\beta_{U}=0.5, \beta_{T}=1.5$ and $M_{\infty}=0$. The dashed curves corresponds to the five combinations: $\mathrm{He}-\mathrm{Ar}, \mathrm{H}_{2}-\mathrm{Ar}$, $\mathrm{Ne}-\mathrm{Ar}, \mathrm{O}_{2}-\mathrm{Ar}, \mathrm{N}_{2}-\mathrm{Ar}$; the solid curves corresponds to the same combinations, but interchanged in the free streams. 


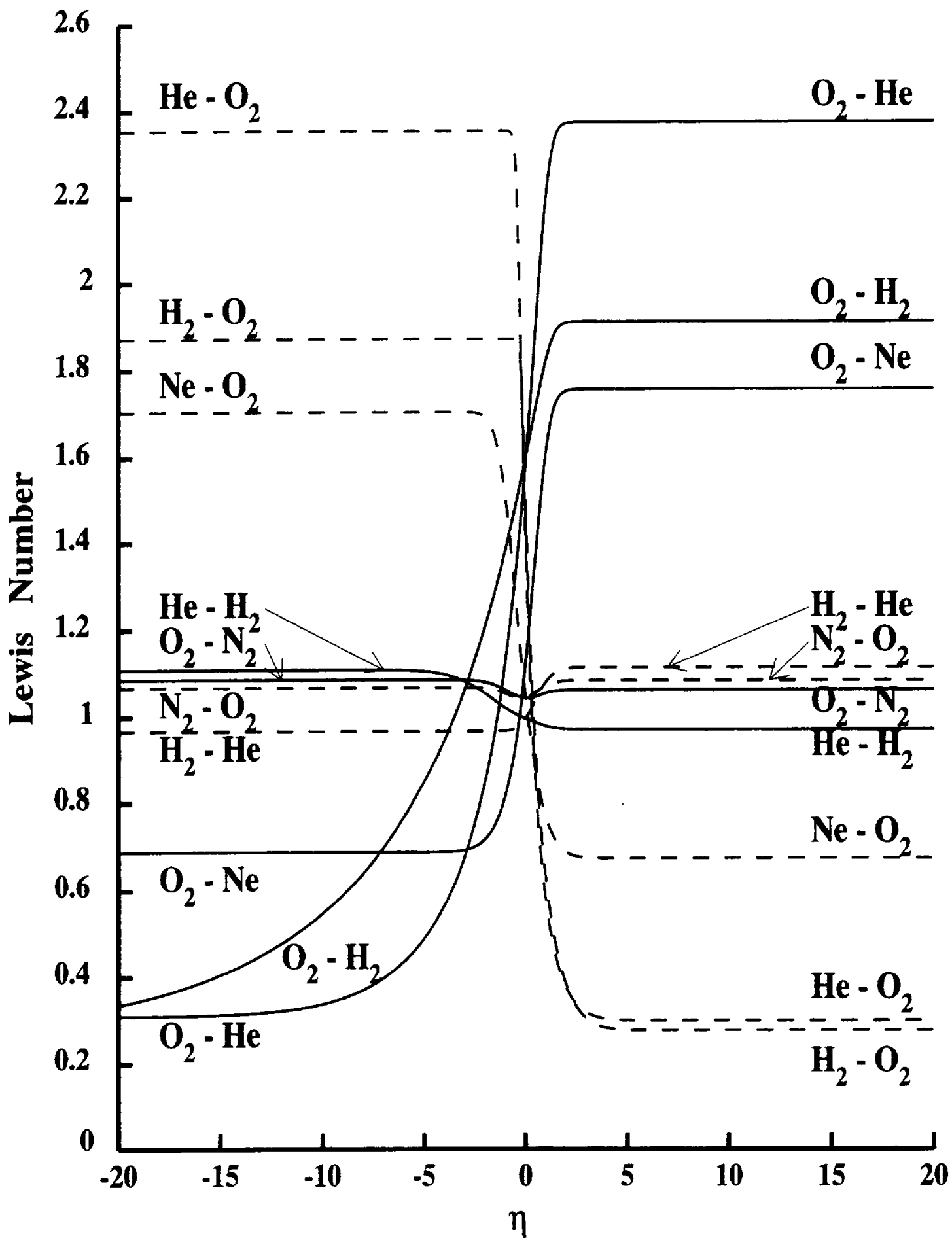

Figure 2. Plot of the Lewis number as a function of $\eta$ for $\beta_{U}=0.5, \beta_{T}=1.5$ and $M_{\infty}=0$. The dashed curves corresponds to the five combinations: $\mathrm{He}-\mathrm{O}_{2}, \mathrm{H}_{2}-\mathrm{O}_{2}$, $\mathrm{Ne}-\mathrm{O}_{2}, \mathrm{~N}_{2}-\mathrm{O}_{2}, \mathrm{H}_{2}-\mathrm{He}$; the solid curves corresponds to the same combinations, but interchanged in the free streams. 


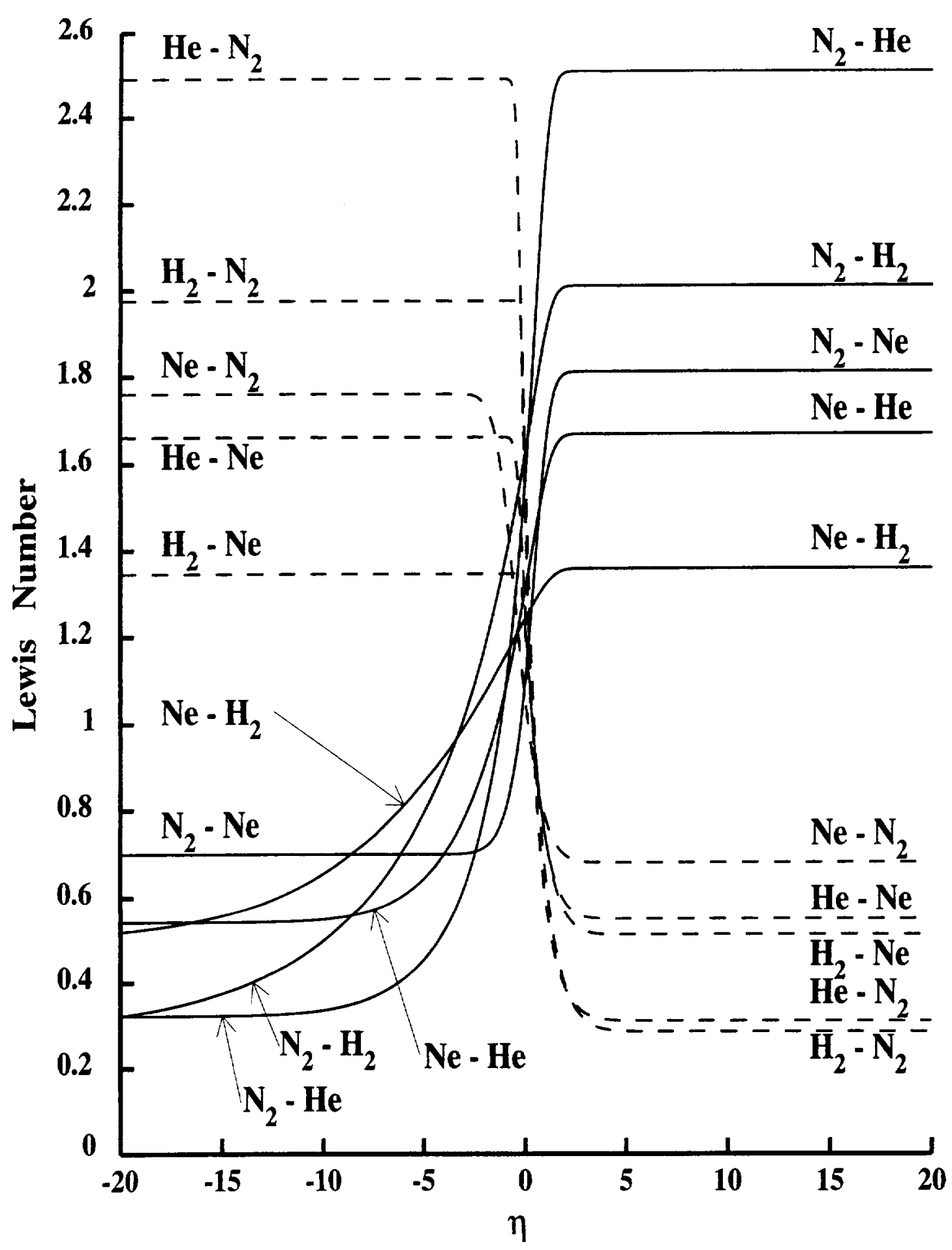

Figure 3. Plot of the Lewis number as a function of $\eta$ for $\beta_{U}=0.5, \beta_{T}=1.5$ and $M_{\infty}=0$. The dashed curves corresponds to the five combinations: $\mathrm{He}-\mathrm{N}_{2}, \mathrm{H}_{2}-\mathrm{N}_{2}$, $\mathrm{Ne}-\mathrm{N}_{2}, \mathrm{He}-\mathrm{Ne}, \mathrm{H}_{2}-\mathrm{Ne}$; the solid curves corresponds to the same combinations, but interchanged in the free streams. 


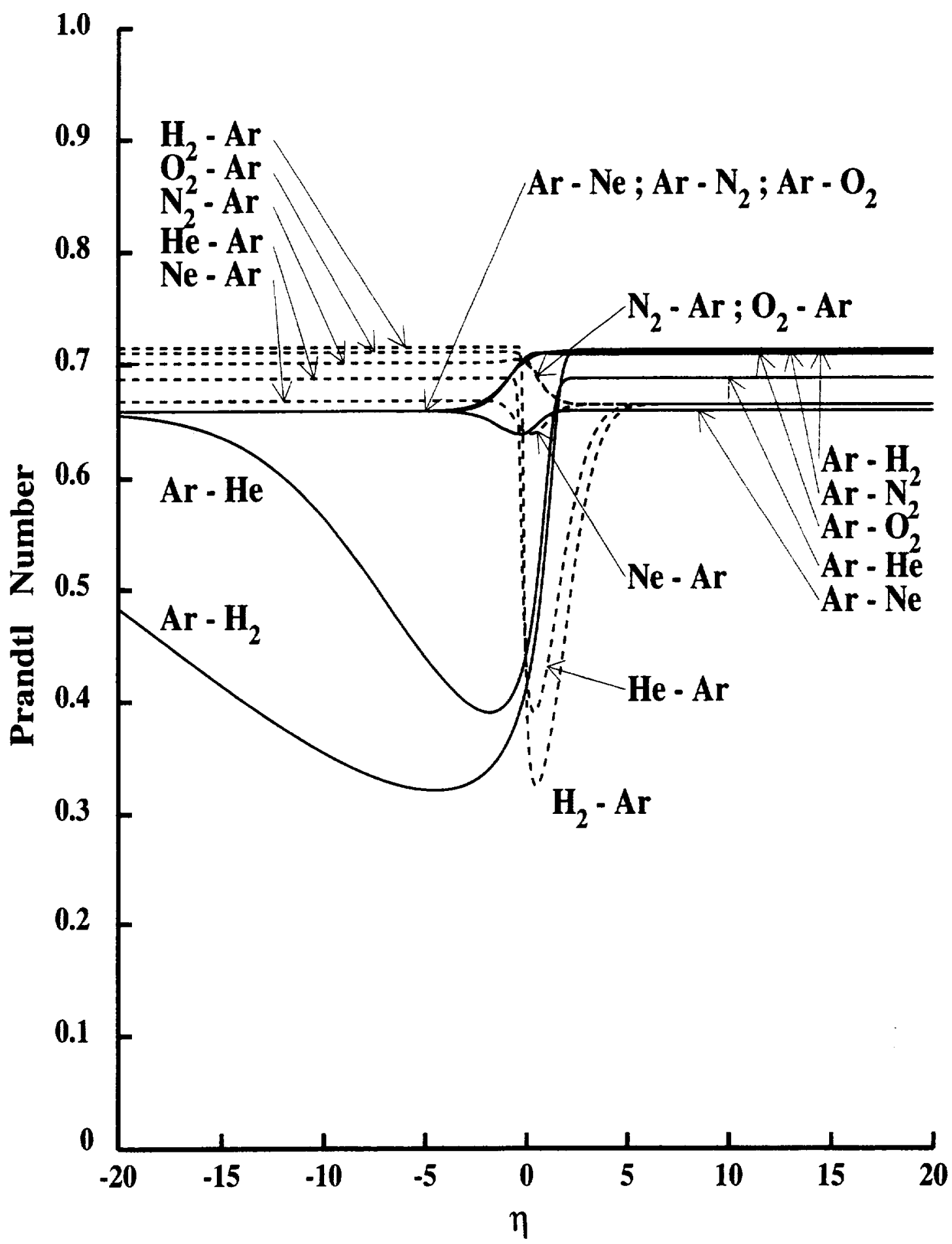

Figure 4. Plot of the Prandtl number as a function of $\eta$ for $\beta_{U}=0.5, \beta_{T}=1.5$ and $M_{\infty}=0$. The dashed curves corresponds to the five combinations: $\mathrm{He}-\mathrm{Ar}, \mathrm{H}_{2}-\mathrm{Ar}$, $\mathrm{Ne}-\mathrm{Ar}, \mathrm{O}_{2}-\mathrm{Ar}, \mathrm{N}_{2}-\mathrm{Ar}$; the solid curves corresponds to the same combinations, but interchanged in the free streams. 


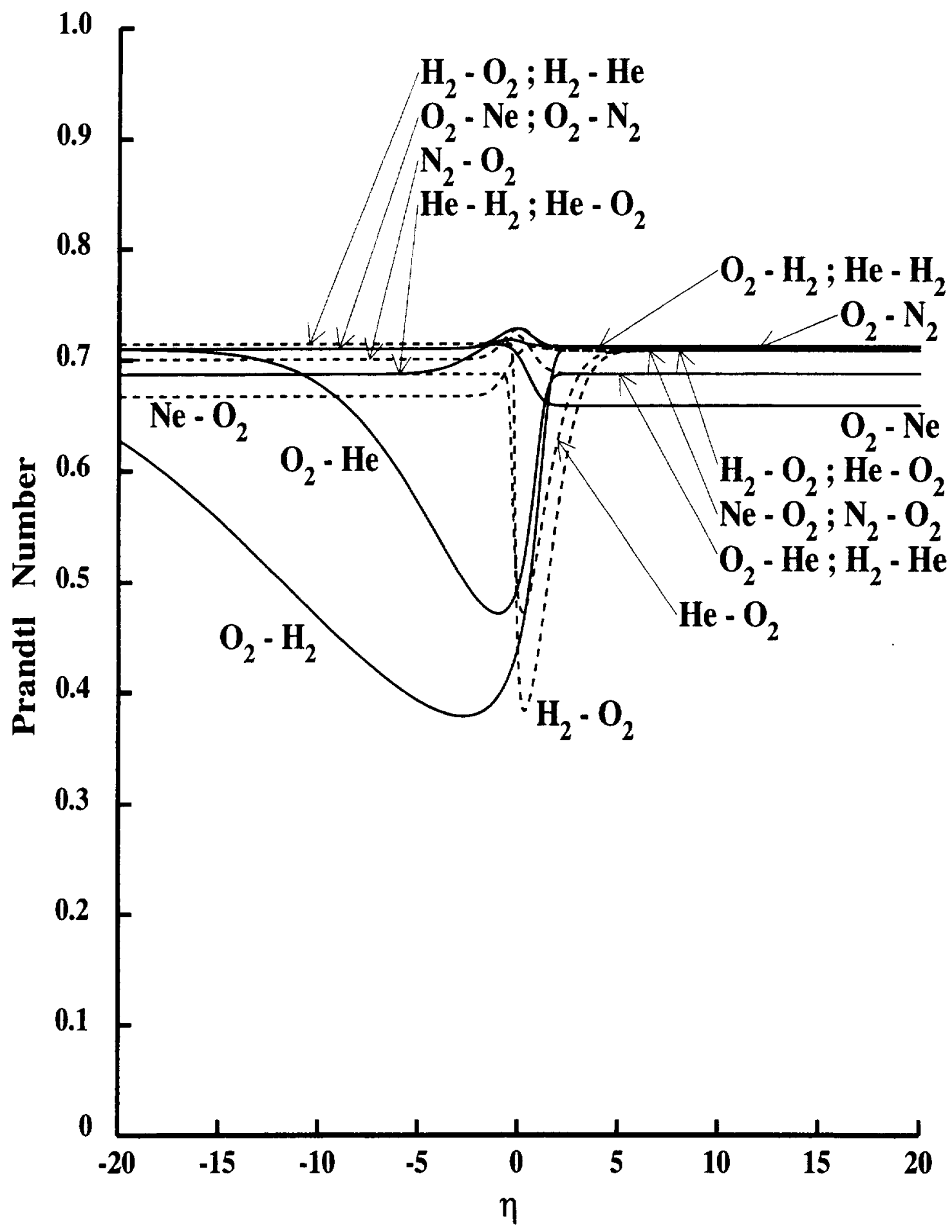

Figure 5. Plot of the Prandtl number as a function of $\eta$ for $\beta_{U}=0.5, \beta_{T}=1.5$ and $M_{\infty}=0$. The dashed curves corresponds to the five combinations: $\mathrm{He}-\mathrm{O}_{2}, \mathrm{H}_{2}-\mathrm{O}_{2}$, $\mathrm{Ne}-\mathrm{O}_{2}, \mathrm{~N}_{2}-\mathrm{O}_{2}, \mathrm{H}_{2}-\mathrm{He}$; the solid curves corresponds to the same combinations, but interchanged in the free streams. 


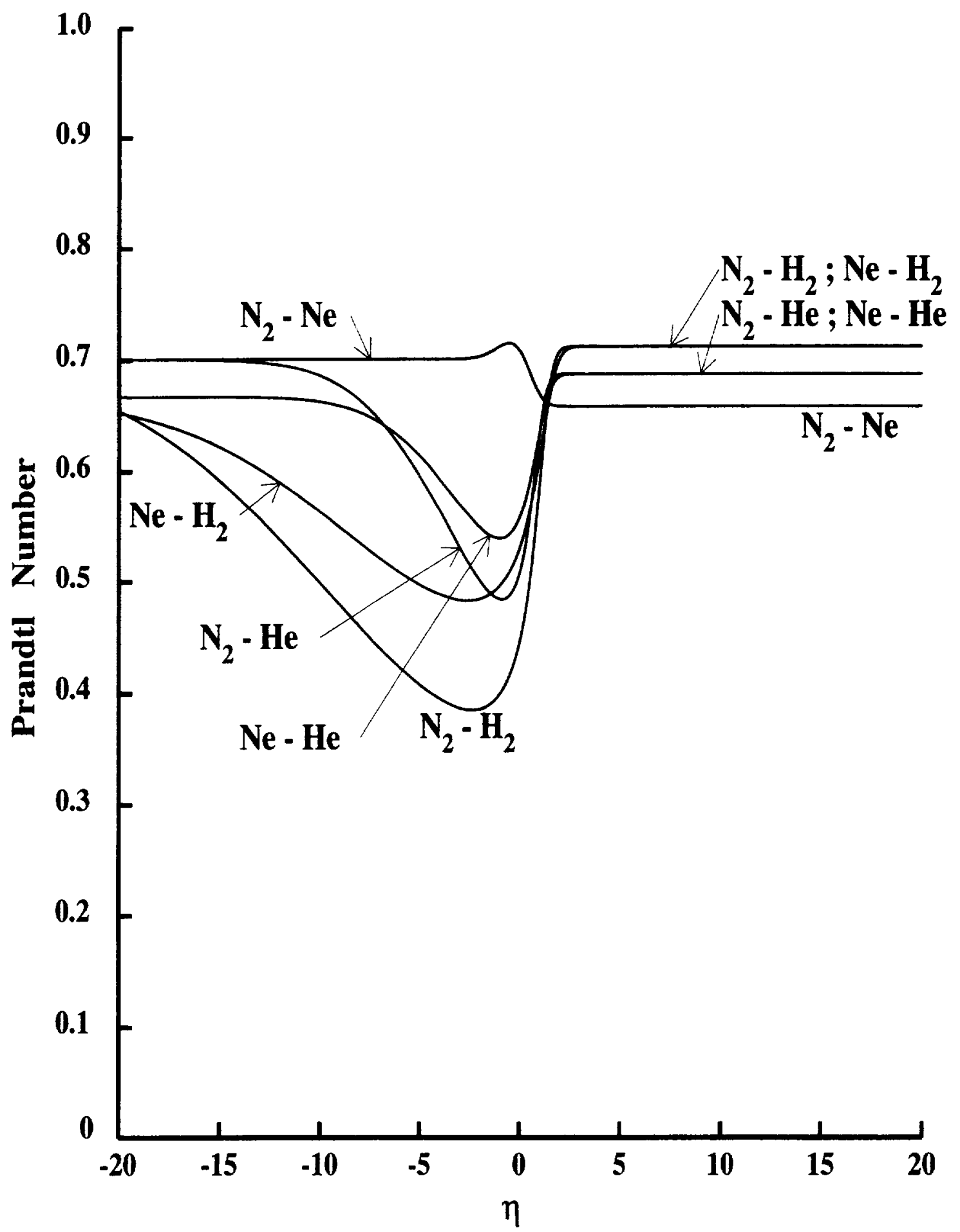

Figure 6(a). Plot of the Prandtl number as a function of $\eta$ for $\beta_{U}=0.5, \beta_{T}=1.5$ and $M_{\infty}=0$. The curves corresponds to the five combinations: $\mathrm{N}_{2}-\mathrm{He}, \mathrm{N}_{2}-\mathrm{H}_{2}$, $\mathrm{N}_{2}-\mathrm{Ne}, \mathrm{Ne}-\mathrm{He}, \mathrm{Ne}-\mathrm{H}_{2}$. 


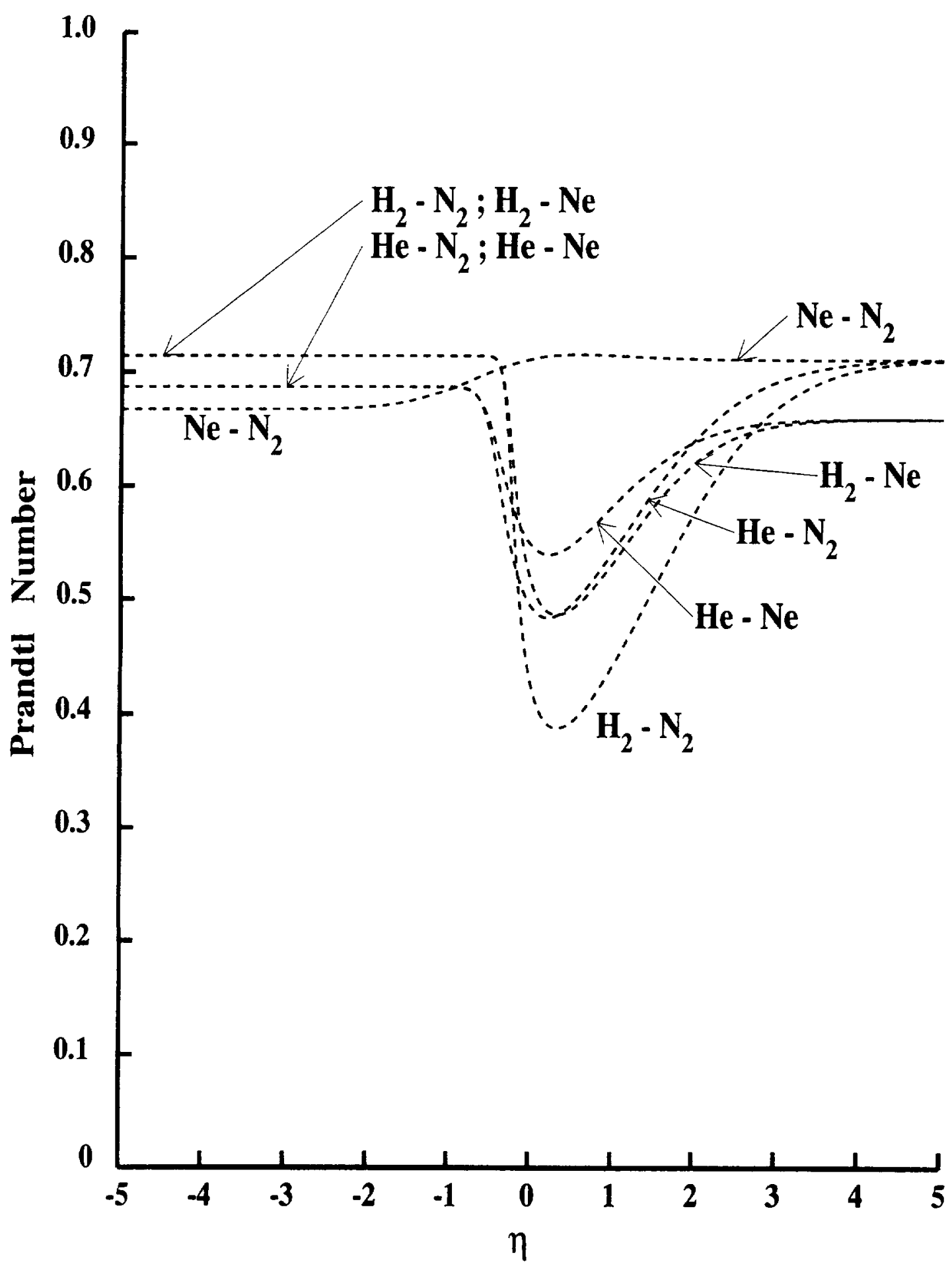

Figure 6(b). Plot of the Prandtl number as a function of $\eta$ for $\beta_{U}=0.5, \beta_{T}=1.5$ and $M_{\infty}=0$. The curves corresponds to the five combinations: $\mathrm{He}-\mathrm{N}_{2}, \mathrm{H}_{2}-\mathrm{N}_{2}$, $\mathrm{Ne}-\mathrm{N}_{2}, \mathrm{H} \epsilon-\mathrm{Ne}, \mathrm{H}_{2}-\mathrm{N} \epsilon$. 

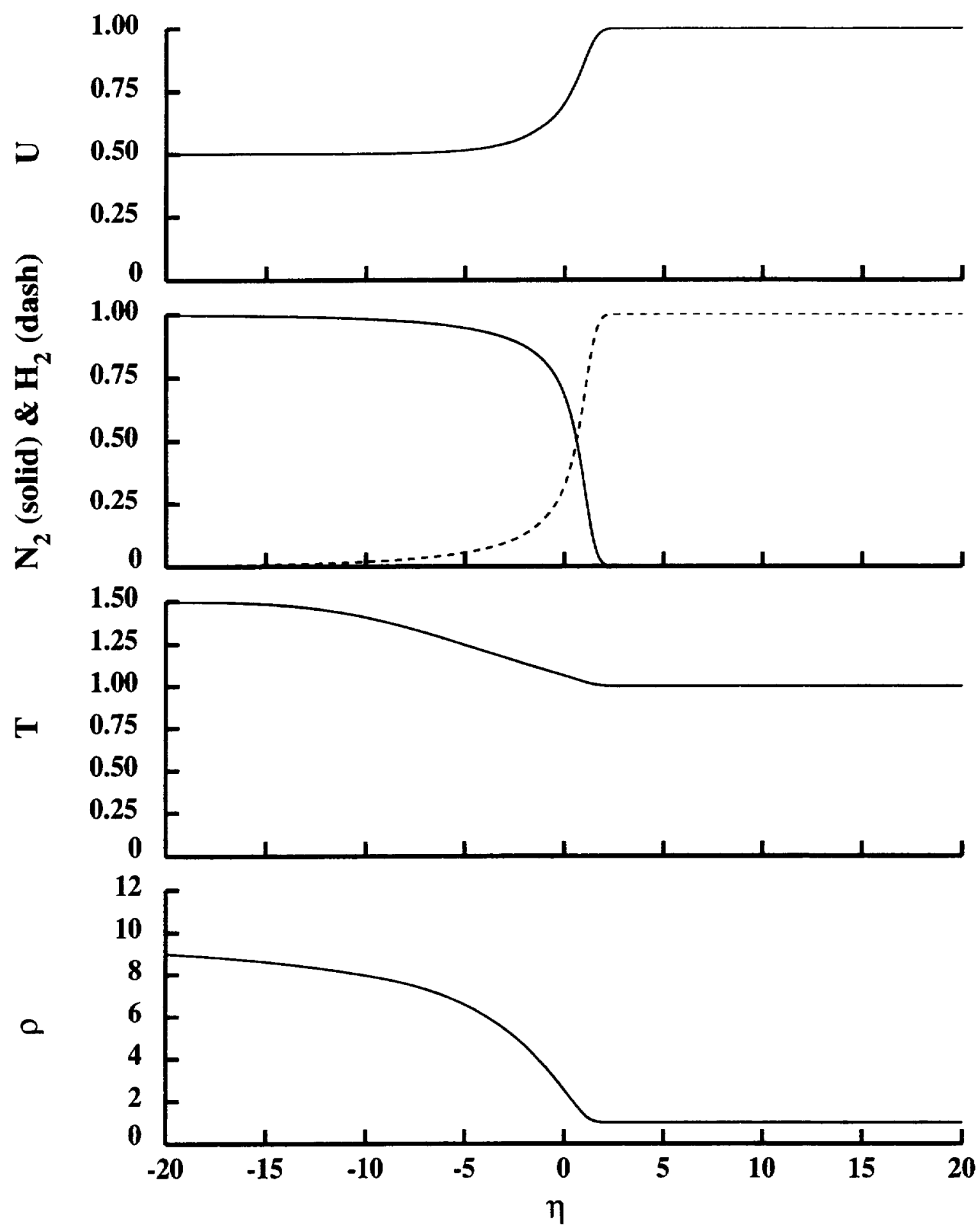

Figure 7. Plot of velocity, mass fractions, temperature and density as a function of $\eta$ for the binary system $N_{2}-H_{2}$. Here, $\beta_{U}=0.5, \beta_{T}=1.5$ and $M_{\infty}=0$. 

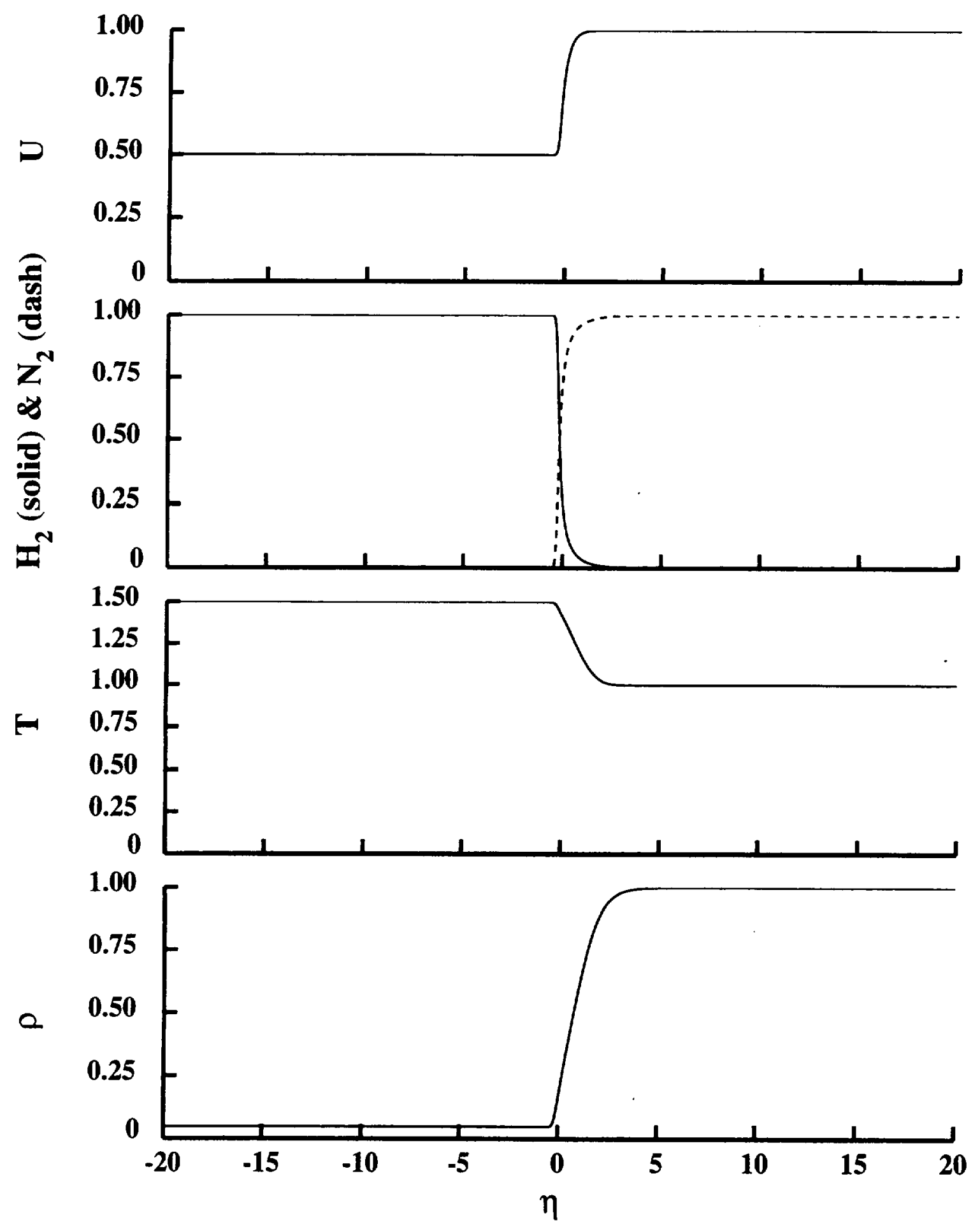

Figure 8. Plot of velocity, mass fractions, temperature and density as a function of $\eta$ for the binary system $H_{2}-N_{2}$. Here, $\beta_{U}=0.5, \beta_{T}=1.5$ and $M_{\infty}=0$. 

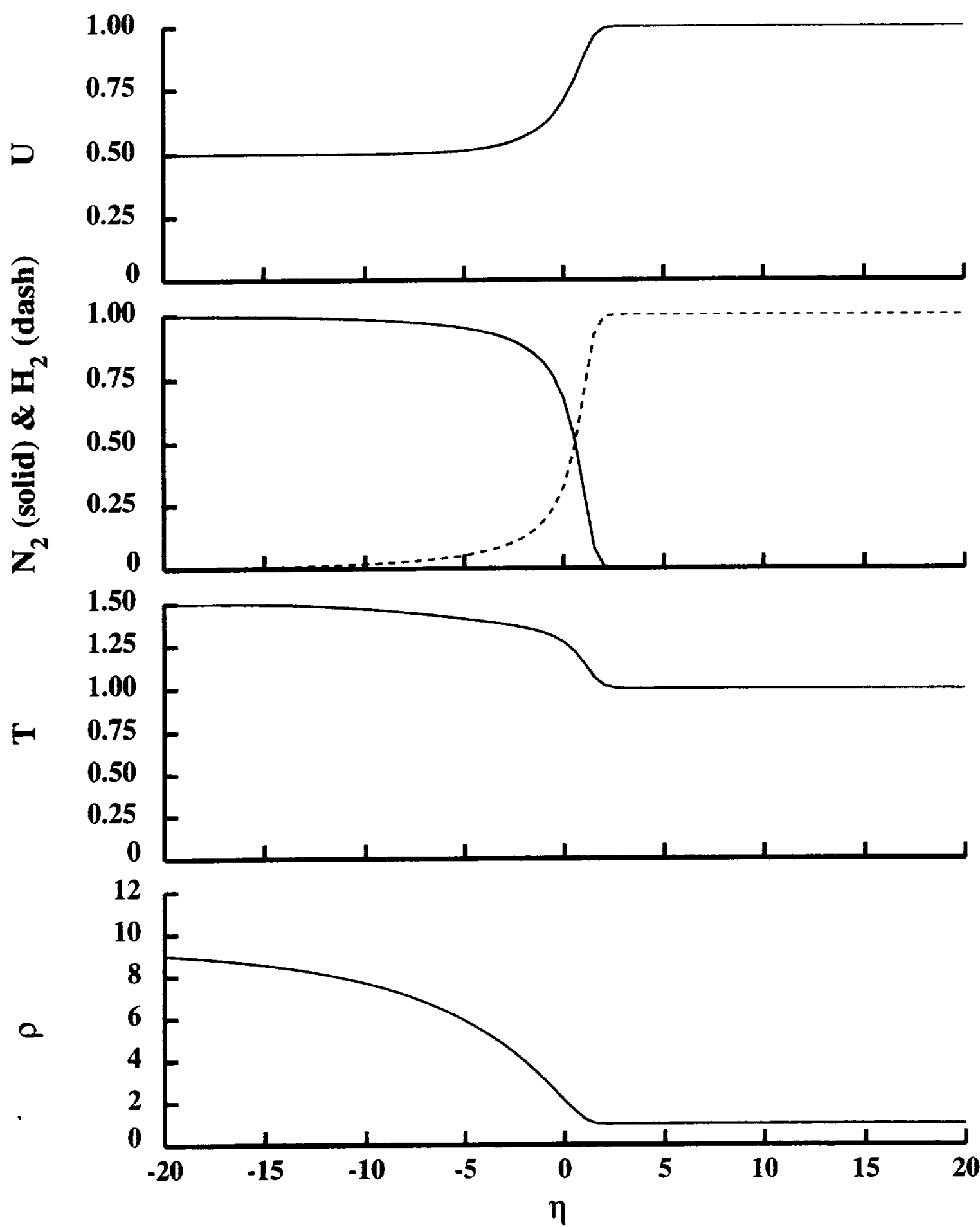

Figure 9. Plot of velocity, mass fractions, temperature and density as a function of $\eta$ for the binary system $N_{2}-H_{2}$. Here, $\beta_{U}=0.5, \beta_{T}=1.5$ and $M_{\infty}=3$. 


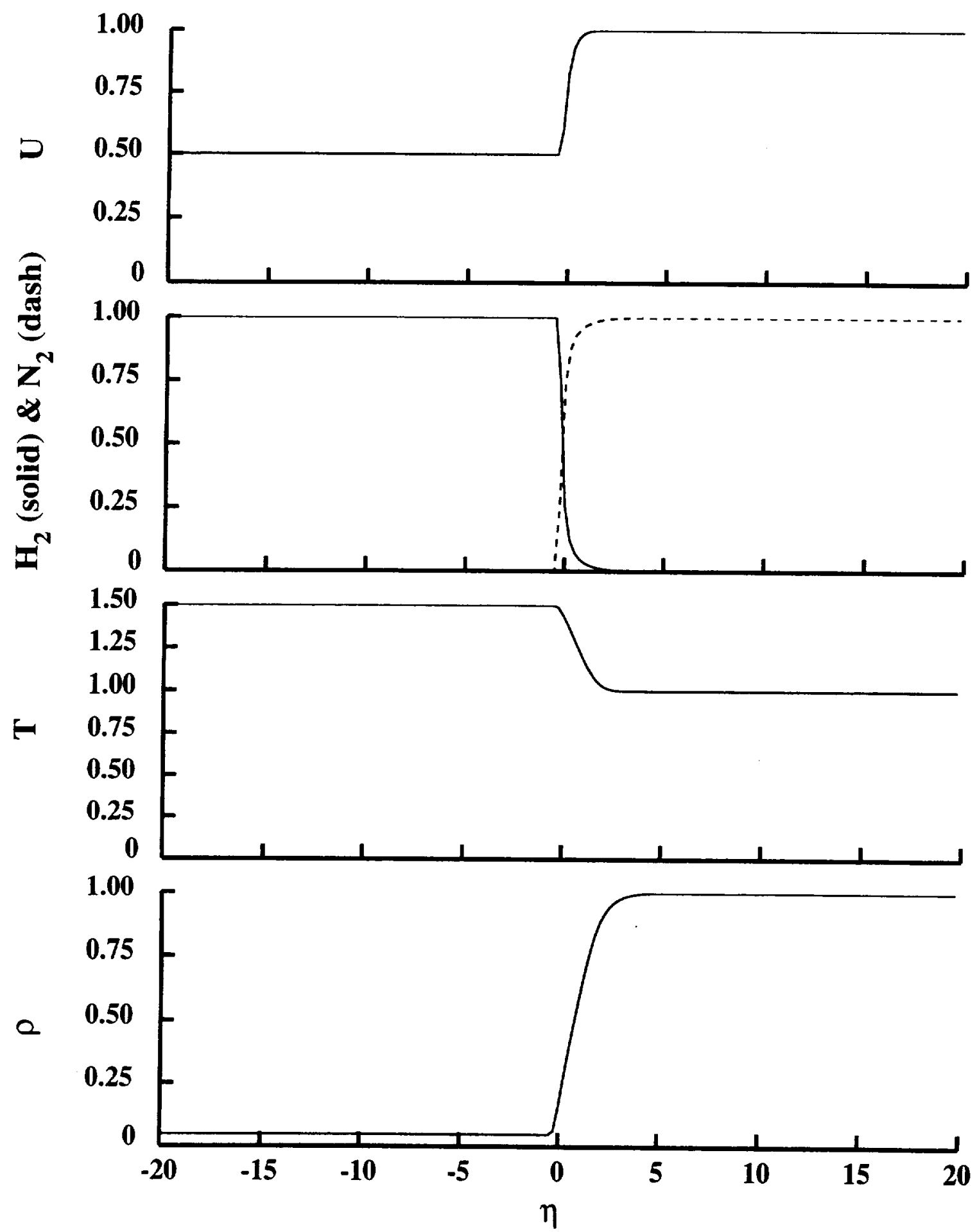

Figure 10. Plot of velocity, mass fractions, temperature and density as a function of $\eta$ for the binary system $H_{2}-N_{2}$. Here, $\beta_{U}=0.5, \beta_{T}=1.5$ and $M_{\infty}=3$. 


- 


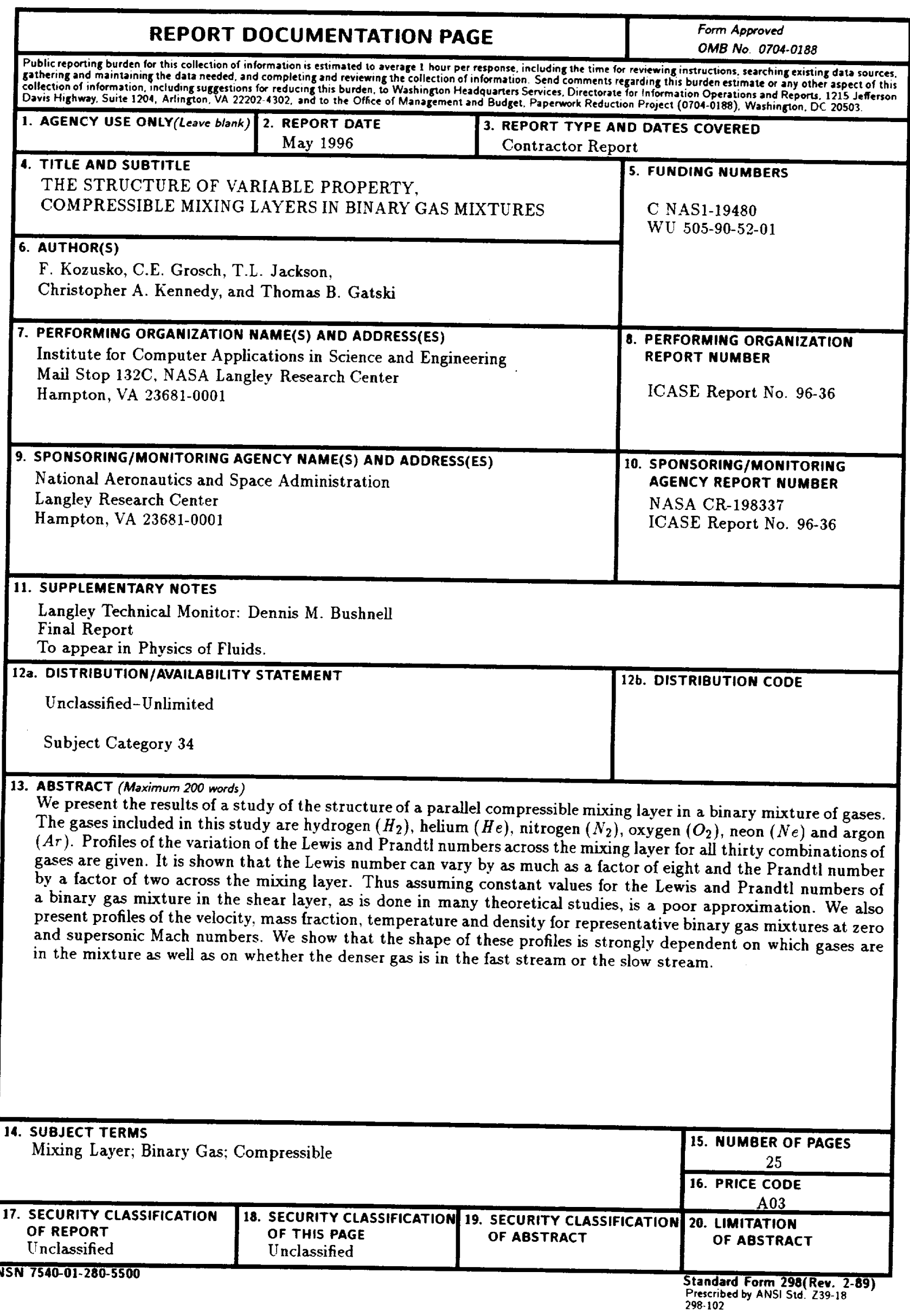

